attached to the teaching of the methods of performing tremendous operations on the abdomen and brain, most of which really save very few lives, and which not one man in 500 who learns about them will ever perform. What an amount of useless biology, anatomy, physiology, and materia medica is stuffed into the student, and simply consumes valuable slices of time out of his five years' curriculum. And yet here is the important subject of anæsthetics, which every student will in due time have to administer, practically passed over as a thing of little consequence, so that many a man gets a diploma who has never once given ether or chloroform. It is time that the schools began to take hold of this matter and to show a practical interest in it, so that the bodies which grant degrees and diplomas may eventually compel the teaching of anæsthetic administration as a necessary part of the student's education.

How is this to be carried out? As regards all hospitals I cannot say, but with regard to those connected with medical schools, I should think that there were very few which at the present day have not an anæsthetist attached to them, and he should be the man to teach the students, every one of whom should be certified by him as having given an anæs thetic to a certain number of persons under his supervision. And, the labourer being worthy of his hire, he should be entitled to a reasonable fee for this. I do not believe in the cry against increasing the expense of medical education. As regards mere fees paid to teachers these are of a most moderate and well-earned character. They amount to little nore than half the sums demanded for admission into most of the important businesses and professions; so that my view is that the anæsthetist should do practical teaching in his own important branch and should be properly paid for so doing. I know well that this view has been very ably advanced in the medical press by certain men of eminence who have this subject at heart, and one of the objects of this little paper is, if possible, to give what help it may to their efforts.

\section{ULCERATION OF THE GESOPHAGUS AND STOMACH DUE TO SWALLOWING STRONG HYDROCHLORIC ACID :}

LESSONS OF TREATMENT DEDUCED FROM THREE CASES.

\section{BY C. B. KEETLEY, F.R.C.S. ENG.}

SURGEON TO THE WEST LONDOY HOSPILAL.

THE main conclusion I draw from a study of these three cases is that in cases of poisoning by the more powerful corrosive acids surgical intervention should be almost immediate and that it is a mistake to postpone resort to surgery until there is no other alternative except that of letting the patient die from inanition. I will give the histories briefly and then discuss the question of treatment.

CASE 1. Enormous dilatation of the stomach developed after accidental poisoning by strong hydrochloric acid, Loreta's operation; complete relief, apparently lasting; rapid recovery of flesh and strength. - In the middle of October, 1897, I was asked by my colleague, Dr. J. B. Ball, to see a female patient of his, aged 32 years, who had eight months previously swallowed pure hydrochloric acid by mistake. It was the most striking case of dilatation of the stomach that I have ever seen and it is much to be regretted that no photograph was taken. When the patient stood up, whether she was viewed from the front or from the side, the whole anterior abdominal wall, except in the left iliac region and the extreme right of the right lumbar region, could be seen pushed forward by the stomach. She was very emaciated and the shape and movements of the organ could be seen with ease. There was a difficulty in swallowing and immediate vomiting, which, together with the history of corrosive acid poisoning, suggested either stricture or spasm of the œsophagus as well as of the pylorus. In fact, at this time it seemed almost equally difficult to get food into and out of the stomach.

On Oct. 18th an operation was performed. An incision was

I A paper read before the Royal Medical and Chirurgical Society on Nov. 12th, 1901 made in the middle line above the umbilicus. "The stomach presented. The pylorus could be felt some inches away in the right iliac or lower part of the right lumbar region. No adhesions or signs of thickening of the stomach wall were found. The pyloric pcrtion of the stomach was now "hauled" outside. The word "haul" gives a better idea of the length and size of the organ than would the usual word "pull." Protective gauze packing was employed. A one-and-a-half inch incision was made into the stomach. The pylorus was thickened and was so contracted that it would only just admit the closed blades of a pair of polypus forceps. Gradual dilatation was made, first with the forceps, next with the little finger, and lastly with a three-bladed rectal dilator. With the last the pyloric opening wa stretched to a circumference of four and a half inches, and a slight sensation of tearing was felt. Closure of the opening and removal of the dilator were next effected and the stomach was washed out with hot water through the wound. The opening in the stomach and the wound in the abdominal wall were then sutured.

Improvement began at once and progressed rapidly. Indeed, as soon as the patient had fully recovered consciousness after the anæs thetic she felt well and complained of nothing afterwards but an inordinate appetite. Vomiting ceased and the patient rapidly put on flesh. A year afterwards she was in good health and strength. The stomach never quite returned to its normal size. Last year I heard that she had not been so well recently, but I did not learn what was the matter. She has left her former address and I cannot find her, so I cannot report on her present state. The patient was shown at a meeting of the West London Medico-Chirurgical Society in December, 1897. I cannot remember who was the medical friend who saw her last year; but if he sees this I hope he will communicate with me.

CASE 2. Suicidal poisoning by strong hydrochlorn acid; rapid development of bronchitis and obstruction to breathing; extreme weakness; abdominal incision, but the stomach not opened on account of sudden collapse ; great teniporary improvement for 10 days; death three days later ; contraction of the pylorus and pneumonia.-The patient, a man, aged 25 years, was readmitted into hospital on Oct. 19th, 1897, the day after the operation on the patient in Case 1. He had been first admitted under the care of Dr. Hood. 32 days before his first admission he had swallowed strong hydrochloric acid with suicidal intent. After passing 18 days in the hospital he was discharged. He had then no pains, there were no physical signs of illness, and he "could swallow thin foods and milk." Very shortly after leaving he began to lose flesh, found difficulty in swallowing even liquids, and at last "what food he did swallow was vomited." When he was readmitted there was a "constant feeling of sickness" and the patient was emaciated. There was no vlceration or cicatrisation of the mouth or the fauces. He could not swallow saliva. Attempts to pass cesophageal bougies caused much distress and induced vomiting. There were signs of pyloric obstruction. The greater curvature of the stomach descended an inch below the umbilicus. Palpation caused slight pain and excited spasmodic contraction. No thickening could be felt. The vomit for the most part was liquid and very dark brown. Constipation was present. On Oct. 21st rectal feeding was commenced. On the 28th when I saw the patient, as he had been getting more and more emaciated, I recommended operation. I noticed that he was then suffering from some bronchial or pneumonic affection with expectoration of copious blackish phlegm.

On Oct. 29th operation was proceeded with. I made an incision to the right of the middle line. The pylorus was exposed, but before further steps could be taken the patient became collapsed and blue. It was considered necessary to postpone opening the stomach. Iodoform gauze (wrung out in 1 in 2000 sublimate lotion) was placed in the wound so as to prepare for a future second stage of operation, without anæsthetic, in a day or two. A few ounces of neutral saline were injected subcutaneously into the axilla and 14 ounces of warm milk into the rectum. A urethral bougie was passed down the cesophagus. My intention, as may have been inferred, was to enlarge the pylorus without a general anæsthetic about 48 hours after the unfinished operation, but a curious change in the patient prevented me. "He was much better in the night and for the first time for eight days was able to take fluid by the mouth." For the next 10 days he took milk fieely and improved in strength and spirits every day. But unfortunately on the e'eventh day a good deal of pain was complained of in the right side. On the 
thirteenth day the note is : "Better night. Kept expectorating dark-coloured phlegm. Very collapsed in morning. Very little pulse. Gradually sank and died." 'The ternperature had ranged from $97^{\circ}$ to $98.4^{\circ} \mathrm{F}$, rising only one degree the day before death.

The only observations noted post mortem were pneumonia of the base of the lung, congestion, and possibly a stricture of the upper part of the cesophagus, thickening and a very tight stricture of the pylorus, and enormous distension of the stomach.

It is difficult to be sure of what occurred in this case after the incomplete operation. Possibly the passage of the urethral bougie down the cesophagus restored the power of swallowing, but as the pyloric stricture remained unrelieved less fluid passed out of than into the stomach, and so the gastric dilatiation increased and the general condition became more dangerous. I was lulled into a false sense of security by the improvement in the patient's spirits and appearance. (The notes of the case were taken by Mr. J. M. Flavelle and Mr. A. Granville. )

CASE 3. Suicidal poisoning by strong hydrochloric acid, stricture and progressive ulceration of the cesophagus and of the pyloric part of the stomach as well as of the pylorus; grent emaciation and depression; gastro-enterostomy with Murphy's button; immediate relief and continued improvenent for nearly six weeks, then death from bronchitis and pneumonia; Murphy's button found in the stomach, and the ulceration of the resophayus unhealed. - The patient, a female, aged 46 years, was admitted under the care of Dr. Hood on Sept. 13th, 1900. When under the influence of drink she had attempted suicide with strong hydrochloric acid. She had "spat out most of it." The mouth and the fauces were burnt by the acid. There were pain all down the throat and in the stomach and great thirst. An emetic and then bicarbonate of potash and calcined magnesia were administered. The pulse was 104 and of good volume and tension. For 10 days she had pain and the vomit occasionally contained blood. Compound mixture of bismuth with glycerine of carbolic acid (10 minims), was given internally, and chlorate of potash gargle. The mixture seemed to remove both the pain and the vomiting. Nutrient enemata were employed. On the eighteenth day (Sept. 30th) the mouth and lips had healed. On Oct. 1st milk was given by the mouth. On the 9 th the nutrient enemata were stopped. 'The patient took more by the mouth; there was great hunger. Swallowing, unfortunately, became more and more difficult. Before the 27th (the forty-third day) scarcely even the smallest quantity of liquid could be swallowed. Emaciation and weakness were extreme. The nutrient enemata had been renewed on the $23 \mathrm{rd}$.

On Oct. 27th operative measures were carried out. A median incision was made. The pylorus presented at once in the middle line, but could not be turned out owing to extensive and tough adhesions. The incision was prolonged up to the xiphoid. 'The left rectus and superjacent skin were cut through transversely. Extensive, strong adhesions of the stomach to the omentum, the abdominal wall, and the transverse colon were partly clamped and were all divided or separated. The stomach could then be moved. The pyloric portion was contracted to the shape of a small sausage; the cardiac end was smaller than natural and almost entirely under the left costal margin. An anterior gastro-jejunostomy with a Murphy's button was performed. The bowel and the stomach apertures were tightened round the halves of the buttons by two or three interrupted fine silk sero-muscular sutures. The contracted pyloric part of the stomach was three or four inches long. Its lumen would not admit the tip of the little finger-in fact, it seemed almost impervious. The parietal wound was closed in layers. There was no drain. The patient was very collapsed after the operation. The pulse in the evening was 176. The nutrient enemata were not retained. Milk and hot water (in equal parts) were therefore given by the mouth at once. Five ounces were given-one ounce every quarter of an hour. This was repeated in the evening; there was no vomiting. The patient's condition improved. On the 28th (the day after the operation) the pulse was 112 . Liquid food was retained both by the stomach and by the rectum. On the 30 th the patient was better still; the pulse was 90 . She romited altogether three times in the course of the first 10 days. On the tenth night she retched a great deal. There was no abdominal tenderness. Did the Murphy's button fall back into the stomach at this time and cause the retching? Feeding by the mouth was stopped for 24 hours and then was resumed cautiously with milk and "Valentirue." There was no more vomiting. 'The wound healed. 'The patient increased in strength and cheerfulness. The temperature was normal till Nov. 20th (the twenty-fifth day after the operation), when it began to rise gradually, and on the twenty-nine day it reached $101 \cdot 6^{\circ} \mathrm{F}$; it only once reached $102^{\circ}$ (four-hourly chart). With the rising temperature was noticed a slight cough with mucous expectoration. There was no pain or tenderness in the epigastrium. Some pain over the base of the right lung continued and there were occasional paroxysms of pain in the "left iliac region." Was this caused by the button, which $x$ rays had a week before shown to have probably fallen back into the stomach? There were râles on coughing, but there was no dulness at the right base. On the 24th the mucus was slightly rusty; the chest was tender when percussed. On the 29 th the sputum was offensive, mnco-purulent, and more copious. On Dec. 2nd (the thirty-ninth day after the operation) the patient could not swallow solid food. On the 4th the diarrhœa which had been present for the last three days still persisted. On the 5 th the patient died from collapse.

The post-mortem examination showed a stricture two inches long, scarcely admitting a lead pencil, at the upper extremity of the oesophagus. At the cardiac end was a second stricture, less tight, but with nlceration still active. 'The Murphy's button was free in the cavity of the stomach. 'The ulceration had quite healed. The cicatrised and contracted pyloric end had further contracted longitudinally to about half its length at the date of the gastro-enterostomy (six weeks before). A narrow curved or sinuous passage led through it into the duodenum. The gastro-enterostomy was perfect with a free passage into the distal loop of the jejunum, and a narrower one into the proximal. The extensive adhesions observed at the operation had nearly all disappeared and nothing remained to interfere with free movements of the stomach. The large bronchi were ulcerated and contained foul purulent secretion. There was grey hepatisation of the left lung throughout. Some pneumonia was present at the base of the right lung. (The notes of the case were taken by $\mathrm{Mr}$. Bennett and $\mathrm{Mr}$. O. Inchley.)

\section{Remarks ON THE Three Cases.}

That which was least injured and non-suicidal recovered but passed through a period of illness which if left unrelieved must have had serious consequences. Both suicidal cases were much worse than Case 1 at the time of operation. Besides, in both cases the bronchial trouble began before operation. In Case 2 the following note was recorded the day after the acid was swallowed: "Large mucous râles all over chest, back and front." Two days later there is the note, "Respiration, especially at night, is very noisy and sounds as if it was obstructed." The note on the day of his discharge is, "Lungs practically clear" ; it continues : "No sickness, no dysphagia, no," \&c. Nevertheless, this patient had to be re-admitted in 14 days worse than ever. There is no note about his respitatory organs on re-admission, probably because attention was concentrated on bis serious œsophageal and gastric troubles. The patients in Cases 2 and 3 did not suffer to anything like the same extent as did the patient in Case 1 from gastric dilatation; indeed, the third patient had a stomach much smaller than normal. But they were more seriounly injured in the œsophagus. A careful post-mortem examination of that organ from Case 3 persuades me that its ulceration was still progressive rather than healing, although her death occurred 40 days after operation and 83 days after swallowing the corrosive acid. In both the fatal cases the patient died from septic broncho-pneumonia; I see no reason for attributing this to the operations. In Case 2 nothing was done but the making of a small incision in the abdominal wall. In Case 3 healing was rapid. A post-mortem examination showed the gastro-entero-tomy to be perfect, and not only was there no peritonitis but most of the adhesions seen at the operation, 40 days before, had been absorbed.

I believe the ulceration of the cesophagus or of the pharynx leads to the infection of the air-passages. This may occur directly through the lymphatics, or indirectly through the passage of muco-purulent discharge downwards through a glottis, perhaps itself cedematous or thickened, or otherwise impaired by the action of the acid, not necessarily on the glottis itself, but on parts closely adjacent to it.

At the same time the physical strength and the mental and moral state of such patients are lowered extremely by 
both the causes and the results of the accident. The utmost conceivable depth of "l,wness" is reached by a patient who, as a consequence of swallowing a corrosive acid suicidally, is for a long period neither able to pass food through the œsophagus nor chyme through the pylorus.

\section{The Question of TreatTMent.}

These cases seem to me to teach certain lessons. Conclusions should be drawn cautiously from a short series of only three cases; but the rules which $I$ am going to lay down are indicated by these cases, not only collectively, but individually. It is not, therefore, a mere matter of statistics.

1. The patient should receive no food (either liquid or solid) by the mouth for several weeks-i.e., he should not be fed by the mouth as soon as he can swallow with little or no pain; but oral feeding should be postponed until there is good reason to believe that the injuries have completely healed.

2. When the injuries are serious (and they generally are so) an operation should be performed within a few days of the date of the poisoning-the sooner the better

It must always be a matter of conjecture to determine whether the injuries have healed or not. The only parts of the injured tract visible are the mouth and pharynx, but the pylorus or even the middle of the stomach may be much worse, as, e.g., in Case 3, not to mention the oesophagus. In Case 3 the mouth and lips are noted as having healed on the eighteenth day, but active ulceration of the gullet was found after death on the eighty-fourth day (three months after the accident).

I am afraid that it is rarely safe to assume that a case of this kind is not serious unless it is positively known that only a minute quantity of acid has been swallowed. Case 3 was scarcely a truly suicidal one. The patient got drunk on the "rent money," was scolded by her husband, drank the acid, but spat most of it out again; she was brought to the hospital and made to swallow calcined lime and an emetic. and yet her injuries were terrible. Even when swallowed by pure accident an ounce or more is easily taken into the gullet before the mistake is discovered and most of it passes into the stomach,. running along the lesser curvature till it is stopped by the pylorus or by food already in the stomach.

All the three cases were treated according to what seems to be the usual practice-that is, the patients were allowed to swallow food when they could do it without much diffculty or pain. The patient in Case 2 was fed by the mouth from the very first day. The subject of Case 3 began with milk on the sixth day and took puddings on the fifteenth day. She never got as far as fish or meat. Granting that the patient should not be fed at all by the mouth for several weeks, and that we should only be satisfied with rectal feeding in trivial cases, the severe cases remain to raise the question of operative treatment.

The region most seriously injured is usually the pyloric part of the stomach. The problem for the surgeon's solution is not simple. The choice of operations apparently lies between gastrostomy, duodenostomy, jejunostomy, gastroenterostomy, and a combination of gastrostomy with gastroenterostomy. Gastrostomy does not give rest to the most injured part-namely, the pylorus. Gastro-enterostomy does not rest the cesophagus. Jejunostomy, when properly done, is practically a double operation. Duodenostomy would seem to be the simplest and most straightforward procedure, although it is liable to permit bile to leak out and irritate the skin. All these methods are open to the objection that, in the by no means unlikely event of an oesophageal or a pyloric contraction taking place after all, a secondary operation may have to be done, in addition to one for undoing the first operation. Therefore the indications would most likely be best met by combining a gastrostomy with a gastro-enterostomy, and carrying the gastrostomy tube through the gastro-enterostomy wound for some distance down the efferent loop of the jejunum. This is practically the method recommended by Witzel for an ordinary gastro-enterostomy, except that in the cases I am writing about something more is desirable-viz., an arrangement for washing out and draining the stomach through the gastrostomy wound. This could be obtained either by using a double tube, especially constructed so that the shorter canal opened into the stomach, or, more readily, by passing the long, narrow gastro-jejunal tube through a short, wide gastric tube. If in spite of treatment pyloric contraction should take place this plan provides a gastro-enterostomy ready made. The gastrostomy wound could be closed or kept open, according to the final condition of the cesophagus. In the hands of careful and experienced operators the method would probably be found very safe, as the patient would be operated on while in fair physical condition, and the gastro-jejunal tube should resist the dangers of the "vicious circle." 'These dangers could be further minimised by using Murphy's button, and, should that contrivance fall into the stomach, the gastrostomy wound itself could be enlarged to permit its extraction. Further, the treatment above recommended would reduce to a minimum the danger of infection of the air-passages, through the swallowing or the regurgitation of septic discharge, or of food, either of which might easily find its way through a glottis œdematous and stiffened either by direct injury or by injury to neighbouring parts. In a long series of cases of poisoning by corrosives now and then the glottis is likely to be so severely injured as to demand prompt tracheotomy. This would make it more than ever desirable not to feed by the mouth.

At the post-mortem examination of Case $3 \mathrm{I}$ was struck by the resemblance of the ulcerated bronchi to those of a case in which bronchial infection and gangrene had been caused by a tracheo-øesophageal fistula.

\section{AFTER-TREATMENT.}

Feeding by the gastro-jejunal tube would be commenced at once, in spite even of moderate ether or chloroform vomit ing, should those anæsthetics be used, but gas alone, or with oxygen, would suffice for the operation described, or even local anæsthesia. Local treatment should be given to (1) the mouth and nose, (2) the pharynx and the osophagus, and (3) the stomach

The mouth. - This should be frequently washed out with warm solution of chlorate of potash or warm boric lotion, or with both. Dirty teeth should be cleaned, diseased teeth should be treated with pure carbolic acid or by extraction, and suppurating alveoli should be attended to. If the process is very painful owing to the burning, gas, cocaine, or eucaine should be used. The nasal passages should be attended to if unhealthy; if healthy they should be left untouched.

The pharynx.-The pharynx is said not to be reached by gargles. It should be sprayed frequently with hot boric lotion, and twice a day it should be dusted with a little, not much, iodoform powder through a puff. As soon as the patient can swallow without pain he should be allowed hot water or hot neutral saline ad lib., and be encouraged to take it.

The stomach - The hot water swallowed should be allowed to escape by the short gastrostomy tube, so that it would tend to wash out the stomach also. In addition, after each meal given by the gastro-jejunal tube, the stomach should be washed out with hot water by the gastric tube.

The application in some such way as that above sketched of the principles of surgery to this distressing class of cases would, I believe, greatly reduce their mortality and lessen the permanent injury done to those who more or less recover Grosvenor-street, W.

\section{NOTES OF A SEVERE AND LONG-STAND- ING CASE OF LUPUS TREATED BY THE APPLICATION OF THE $\mathrm{X}$ RAYS.}

BY GEORGE H. RODMAN, M.D. DURH., M.R.C.S. ENG.

THE patient whose treatment is the subject of the following notes is a woman, aged about 33 years, who has suffered from lupus of the face, which is reported to have started after an operation for the removal of a diseased portion of the left malar bone some 20 years ago. There is a history of tuberculosis in the family, one sister having died from phthisis at the age of 14 years, and a brother also was the victim of laryngeal phthisis. The lupus had been scraped about 15 years ago; since then it has been treated medicinally and by the local application of ointments and lotions. At the time of starting the treatment by the $x$ rays, on April 19th, 1901, when Fig. 1 and Fig. 2 were obtained, the disease was involving both sides of the face, the ulceration being continued under the chin. On the left side of the face the disease extended from the zygomatic 